\title{
Focal Dermal Hypoplasia with Uterus Bicornis and Renal Ectopia: Case Report and Review of the Literature
}

\author{
Rocío F. Lopez-Porras $^{a} \quad$ Carlos Arroyo $^{b} \quad$ Elena Soto-Vega ${ }^{c}$ \\ ${ }^{a}$ Hospital General del Sur, bHospital Universitario de Puebla, Benemérita \\ Universidad Autónoma de Puebla, y 'Facultad de Medicina, Universidad Popular \\ Autónoma de Puebla, Puebla, México
}

\section{Key Words}

Focal dermal hypoplasia · Goltz syndrome · Uterus bicornis · Renal ectopia

\begin{abstract}
Focal dermal hypoplasia (FDH) is a rare inherited genodermatosis with an X-linked dominant trait. FDH is associated with skin defects and other abnormalities of bone, nails, hair, limbs, teeth and eyes. We present the case of a 26 -year-old female in the 27th pregnancy week and a previous history of miscarriage. After careful physical examination and dermal biopsy, histopathology revealed that the patient was a carrier of FDH. This is the first report in the literature describing that FDH is associated with uterus bicornis and renal ectopia. Our association could be attributable to early embryonic abnormalities related with $\mathrm{FDH}$ because both the uterus bicornis and the renal ectopia originate around the 3th-6th week of embryonic development. We are unable to confirm that the miscarriages were caused by inherited FDH or that uterus bicornis was the cause. We conducted a literature review using the following terms: FDH, Goltz syndrome, uterus bicornis, and renal ectopia.
\end{abstract}

\section{Introduction}

Since Liebermann reported the first case of focal dermal hypoplasia (FDH) in 1935, there have been several clinical reports of the disease and its various associations.

Liebermann named it 'atrophodermia linearis maculosa et papillomatosis congenitalis'. In 1962, Goltz introduced the term 'focal dermal hypoplasia'. Since then, various names have been proposed for this syndrome, such as Goltz syndrome, Gorlin-Goltz syndrome, and congenital ectodermal-mesodermal dysplasia, etc. [1]. 
FDH is a sporadic genodermatosis, characterized by a dysplasia of multiple tissue derivates from the ectoderm and mesoderm. This disease almost exclusively affects women $(80 \%)$, and in most men it is lethal. The incidence is estimated to be $1: 50,000$ to $1: 150,000$ in the general population [2].

The FDH syndrome involves different organs. One of the main characteristics are patches of skin with linear atrophy, hypo- or hyperpigmentation associated with dermal defects that cause fat herniation, as well as multiple papillomatosis of the mucosal membranes or raspberry mark of the skin, with telangiectasias. These lesions can be associated with laryngeal, esophageal or gastric obstruction and can be confused with condylomas [3,4]. There are reports of newborns with patches of total skin absence [5].

Facial abnormalities involve asymmetry caused by bone hemiatrophy or microcephaly, and low-set ears. The hair is usually sparse and fragile, with areas of alopecia and madarosis. Eye abnormalities include microphthalmia with bilateral coloboma of the iris, ectopia lentis, strabismus, anophthalmia, nystagmus, irregularities of the pupils, corneal defects, photophobia and ptosis, etc. [6]. The nasal bridge tends to be narrow with a broad nasal tip, unilateral notch of the ala nasi and chin accentuation. The oral cavity can have a high arched palate, double lingual frenulum, cleft lip and palate, absence of the labial sulcus, gum hypertrophy and papillomas of the gums, tongue, palate and oral mucosa. Finally, oro-dental malformations consist of agenesis or teeth dysplasia, hypodontia, hyperdontia, microdontia, enamel defects and delayed dental eruption [7-9].

Patients also have systemic osteopenia and low stature, spina bifida occulta, scoliosis in up to $20 \%$ of patients, sloped shoulders, and hypoplasia of pelvic bones. Patients tend to have joint hypermobility, hand and foot asymmetries, syndactyly, ectrodactyly, polydactyly, agenesis or finger hypoplasia, and in extreme cases, lobster claw hand [8]. Xrays show large bones with osteopathia striata. The nails could show dystrophy or agenesis, and partial aplasia cutis.

These patients have pulmonary venous drainage defects and mediastinum dextrorotation. The gastrointestinal malformations described are intestinal malrotation, inguinal, umbilical, epigastric or diaphragmatic hernias and rectal prolapse, and, as mentioned earlier, intestinal obstruction. There are descriptions of kidney and ureter malformations such as horseshoe kidney, hypoplasia or agenesis and bifid ureter. Mental retardation is frequently found [10]. These individuals could have hearing defects. Apocrine gland abnormalities cause hydrocystoma and in some cases anhidrosis. Because of all these malformations patients tend to have frequent infections [11].

Skin histological description shows atrophy of dermal connective tissue which is replaced by fat cells, with partial-to-complete absence of portions of the dermis, loose collagen bundles, collagen fibers with irregular bands, abnormal fibroblasts, and disruption of the basement membrane. The endoplasmic reticulum and Golgi apparatus are nonprominent in the dermal fibroblasts, and the fat cells are multilocular and immature, and there tends to be a disruption of the basal membrane. The abnormal collagen formation correlates with clinical skin atrophy. The papillomatous lesions consist of a fibrovascular stalk composed of loose connective tissue with dilated blood vessels and a variable perivascular infiltrate of inflammatory cells, composed of lymphocytes with numerous dermal macrophages. 


\section{Case Presentation}

A 26-year-old female in the 27th pregnancy week and a previous history of miscarriage presented with high blood pressure $(160 / 120 \mathrm{~mm} \mathrm{Hg})$, phosphenes, tinnitus and no fetal heart beat. Preliminary lab results showed proteinuria, high glucose $(120 \mathrm{mg} / \mathrm{dl})$ and creatinine $(1.6 \mathrm{mg} / \mathrm{dl})$, with a creatinine clearance of $28.1 \mathrm{ml} / \mathrm{min}$ and acute renal insufficiency. Initial diagnoses were HELLP, severe oligohydramnios, associated with pre-eclampsia and probable fetal death.

An abdominal ultrasound was done, and no fetal heartbeat was observed, confirming the diagnosis of intrauterine fetal demise. The ultrasound also showed that the patient presented renal ectopia: both kidneys were located on the right side.

As a consequence of fetal death, the suspicion of HELLP syndrome and pre-eclampsia, the patient underwent an emergency caesarean delivery of a 400-gram male dead fetus. During the surgical procedure, no typical uterus morphology was observed, with two separate uterine horns revealing that our patient had a bicornuate uterus. Unfortunately, the patient did not accept a necropsy of her offspring, and thus there is no further description of the dead fetus.

The patient's phenotype was atypical, with a triangular facial contour, scalp with diffuse alopecia, and total absence of eye lashes and partial alopecia of the eye brows, left hemifacial hypoplasia, low-set ears with hypoplasia of the helix and asymmetry of the ear lobes and inferior skin fusion, flat nose, high arched palate, oral cavity with incomplete cleft palate, microdontia and various dental agenesis, micrognathia and right-sided deviation of the chin (fig. 1). Her skin had areas of irregular hypo- and hyperpigmentation with lenticular and poikilodermal appearance. Her arms had xanthomatous lesions with a verrucous texture. Her thumb nails were absent and the remaining nails had a convex tendency, her 5 th fingers were atrophic, hypoplastic with camptodactyly. The perianal and introitus vaginae had verrucous reddish lesions of $2 \times 7 \mathrm{~cm}$ in size (fig. 2), and her limbs had several vegetal, yellow neoformations of soft texture. Her left foot had hypoplasia on the distal phalanx of the first toe and nail hypoplasia. A verrucous distal lesion of the second toe and cutaneous syndactyly could be seen between the third and fourth toes. Finally, her right foot had a polydactyly on the fifth toe.

The skin biopsy showed a thin dermis, and diminished collagen fibers with infiltration of fat cells throughout the different epidermis layers, a severe sloughing of the corneous layer, as well as hyperplastic and hypoplastic areas of the epidermis, confirming the diagnosis of FDH. There was an absence of the normal invaginations in the germinative and papillae dermal junction, some areas of the skin had hyperpigmentation as a result of melanocytes deposits. Finally, the collagen present in the dermis was lax because of erythrocytes infiltrate (ig. 3 ).

\section{Discussion}

Although there are over 300 cases of FDH in the literature, none of them report abnormalities in the genitourinary tract such as the ones found in our patient (uterus bicornis and renal ectopia). There are six reports of renal agenesis in FDH patients, and there are no reports of renal ectopia; concerning the uterine malformations there is only one report of the hand-foot-uterus association with FDH.

Müllerian duct alterations are generally associated with spinal, cloacal and renal abnormalities, which occur early in their embryogenesis $[12,13]$. The incidence of uterus bicornis is of 1:5,400 newborns, and it occurs due to incomplete fusion of the two Müllerian ducts at the level of the fundus, resulting in a caudal portion of the uterus that is normal and the cranial part that is bifurcated. This anomaly is associated with several obstetrical complications like abortion (36\%), preterm birth (23\%), and normal delivery (41\%). The Müllerian ducts develop in the 6th week from the paramesonephric ducts, and fuse in order to form the uterus. 
The incidence of renal ectopia is of approximately 1:1,000 newborns, and it is due to an abnormal migration of the renal blastema and its attachment to the ureteric bud. This takes place later than the renal formation in the 3th-6th week, and thus the renal migration towards the lumbar fossa occurs between the 6th-9th week.

The abnormalities in our patient involve genetic abnormalities early in the developmental stages. Skin abnormalities appear during the 4th-5th week when the most part of the skin is formed. All of the above-mentioned would explain an accumulation of abnormalities around the 4th-6th week $[14,15]$.

FDH cases are sporadic, but during the patient evaluation, the patient mentioned that her mother's phenotype was the same, presenting with hypo- and hyperpigmentation patches and verrucous lesions.

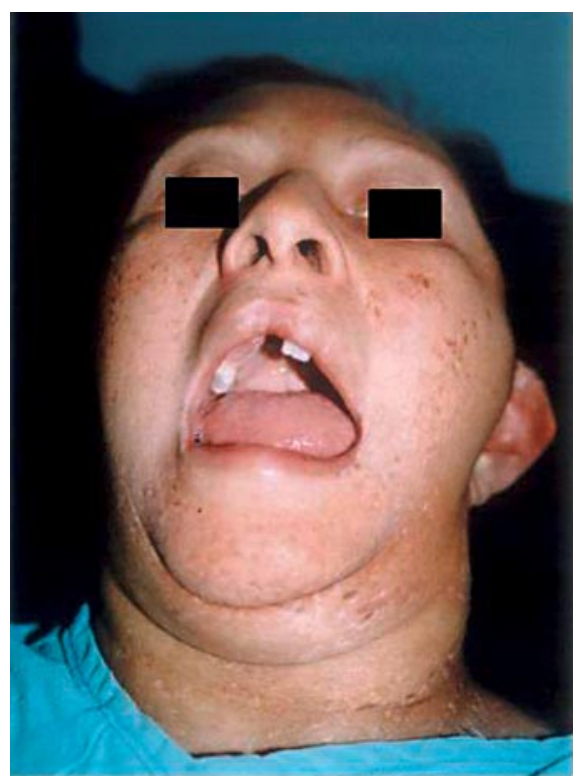

Fig. 1. Patient's face showing marked facial asymmetry, hyperpigmented skin, microdontia and multiple dental agenesis. 


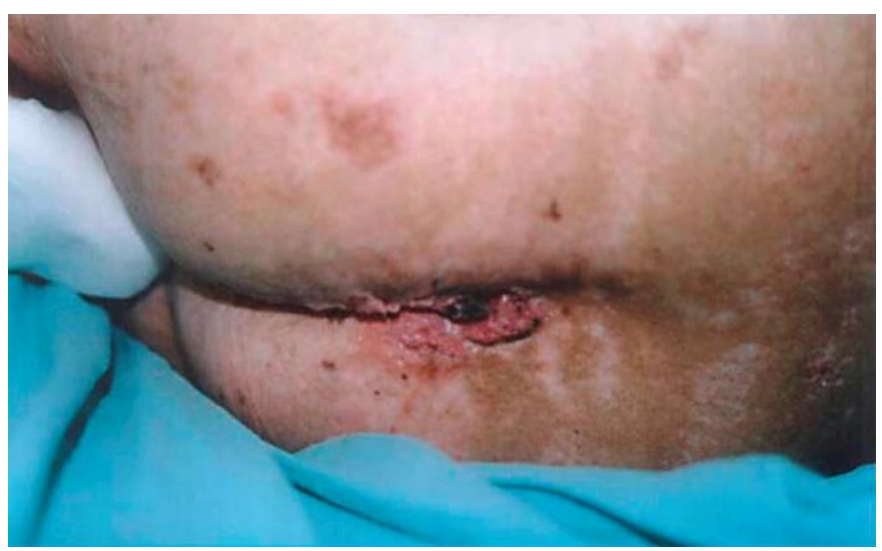

Fig. 2. Perianal verrucous reddish lesions.

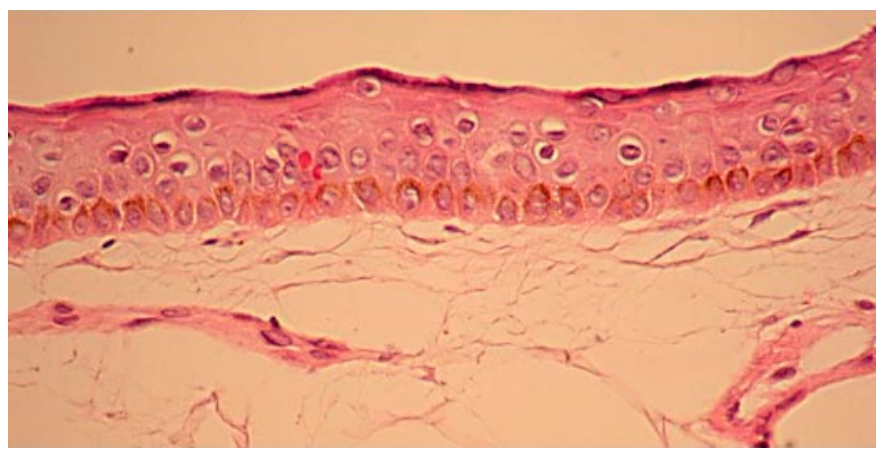

Fig. 3. Atrophy of the epidermis, with subcutaneous fat located in the papillary dermis.

\section{References}

1 Goltz RW, Peterson WC, Gorlin RJ, et al: Focal dermal hypoplasia. Arch Dermatol 1962;86:708-717.

2 Riyaz N, Riyaz A, Chandran R, et al: Focal dermal hypoplasia (Goltz syndrome) Indian J Dermatol Venerol Leprol 2005;71:279-281.

-3 Maymi MA, Martín-García RF: Focal dermal hypoplasia with unusual cutaneous features. Pediatr Dermatol 2002;24:387-390.

4 Sacoor MF, Motswaledi MH: Three cases of focal dermal hypoplasia (Goltz syndrome). Clin Exp Dermatol 2005;30:35-37.

5 Mianda SB, Delmaestro D, Bertolin R, et al: Focal dermal hypoplasia with exuberant fat herniations and skeletal deformities. Pediatr Dermatol 2005;22:420-423.

-6 Prenner JL, Ciaccia S, Capone A Jr, et al: Retinal detachment in focal dermal hypoplasia. Eur J Opthalmol 2004;14:166-168.

7 Al-Ghamdi K, Crawford PJ: Focal dermal hypoplasia - oral and dental findings. Int J Paediatric Dent 2003;13:121-126.

-8 Ogunbiyi AO, Adewole IO, Ogunleve O, et al: Focal dermal hypoplasia: a case report and review of literature. West Afr J Med 2003;22:346-349.

\$ Ljubenović M, Ljubenović D, Binić I, et al: Gorlin-Goltz syndrome. Acta Dermatovenol Alp Panonica Adriat 2007;16:166-169. 
10 Casaroto AR, Rocha Lourdes DC, Moreschi E, et al: Early diagnosis of Gorlin-Goltz syndrome: case report. Head Face Med 2011;25:2-9.

11 Tenkir A, Teshme S: Golz Syndrome (focal dermal hypoplasia) with unilateral ocular, cutaneous and skeletal features: case report. BMC Ophthalmol 2010;10:28-35.

12 Heinonen PK: Unicornuate uterus and rudimentary horn. Fertil Steril 1997;68:224-230.

13 Rackow BW, Arici A: Reproductive performance of woman with Müllerian anomalies. Curr Opin Obstet Gynecol 2007;19:229-237.

14 Schedl A: Renal abnormalities and their developmental origin. Nat Rev Genet 2007;8:791-802.

15 Miyazaki Y, Ichikawa I: Ontogeny of congenital anomalies of the kidney and urinary tract. CAKUT. Pediatr Int 2003;45:598-604.

Rocío F. Lopez-Porras and Carlos Arroyo contributed equally to this work. 